\title{
Chitosan Matrix: Own Unequivocal Myriad Utility in Modern Scientific Development
}

\author{
Rajendra S Dongre* \\ Department of Chemistry, RTM Nagpur University, India \\ *Corresponding author: Rajendra S Dongre, Department of Chemistry, RTM Nagpur University, India \\ Submission: 制 November 07, 2017; Published: 眥 January 23, 2018
}

\begin{abstract}
Chitosan is an amino polysaccharide $2^{\text {nd }}$ abundant biopolymer after cellulose is most $\mathrm{R} \& \mathrm{D}$ explored in diversified fields like biology/biochemistry, organic/polymer chemistry, pharmacology and medicines as being remarkably reported numerous publications/patents. Chitin/chitosan matrix is facile to physico-chemical modifications, in highly sophisticated functions resulting new biocomposites owing greater possibilities widespread over cellulose. Purity/quality condition of this chitosan offered as high, mid and industrial grade that's facile for formulation and to provide solutions of problems in varied fields due to unique parameters viz; bio-compatibility, non-toxicity, anti-microbials and hemeostatic agents. This mini review describes the suitable formulated/fabricated/altered products like biomarkers, biosensors, quantum dots etc, with adoptable higher productive utility of chitin/chitosan chemistry in nano-science and technology.
\end{abstract}

\section{Introduction}

Science and Technology are essential for global growth/ developments that are invariably altered our lives in view of connectivity, communication and transactions, besides economic progress, as they strengthen advances, improvements in health, education and infrastructure. Twenty first century technological most remarkable revolutions includes viz; micro-processor, telecom, satellites, and bio/nano-technology interaction of insights and applications arising when these technologies converge. Scientific innovations and technological throughput creatively drive/boost varied fields like health science, environments, technological growth and livings by enabling affordable/accessible services, myriad reliable products in education and healthcare. The quest of new chemicals is ever from ancient times, prevalently polymeric composites own pivotal status in material science with regards to characteristics performance, prospective applications besides diversity, novelty and versatility as devoid in other materials. The promise of composites lies in their multi-functionality; novelty and versatility, besides possibly realising unique combinations are unachievable in conventional materials.

Chitin/chitosan (amino polysaccharide) is less advanced than cellulose with respect to R\&D and utility, still noticed in many fields like biology/biochemistry, organic/polymer chemistry, pharmacology and medicines as being remarkably reported numerous publications/patents. Chitin skeleton is facile to physico-chemical modifications, thus actively explored in highly sophisticated functions and resulted into new bio-polymeric composites owing greater possibilities widespread over cellulose in future. Chitin/chitosan is categorized by purity/quality into high, mid, and industrial grade polymeric skeletons that's facile to formulate to get solutions of problems in varied fields due to unique parameters viz; bio-compatibility, non-toxicity, anti-microbials, hemeostatic agents and more. Suitable formulating technique adoptions lead to higher productivity and wide utility of chitin/ chitosan chemistry in science and technology [1].

Shrilk is biodegradable plastic yields from shrimp shells derived chitins acts as silk protein substitute to planet-clogging plastics besides implantable medical devices. Wyss Institute scientists developed biodegradable shrilk: insect cuticle's skeleton mimic a laminated silk fibroin which own myrid utilities like in composts/ fertilizers to release nitrogen-rich nutrients, FDA-approved devices, to create implantable foams, films and scaffolds for surgical closure, wound healing, tissue engineering, and in regenerative medicines. Today, synthetic plastic are produced @ 300 million tons/year with only $3 \%$ recyclability and remaining $97 \%$ breaks down in oceans/landfills harming the green sphere/environment. However, numerous bioplastics are derived from cellulose polysaccharide, still unable to fabricate 3D complex shapes to offer the conventional hardiness of plastics. Wyss Institute's shrilk is tough and responsible for the requisite hardness available for licensing.

Biotechnology is multidisciplinary interfaces between science, engineering and technology branches. Nano-biotechnology exploits nanotechnology for improving biotechnology; whereas bio-nanotechnology fabricates natural/biomimetic benefits to yield special nano-structures thus both fields are complementary 
however, untapped field of science. Nano-science/technology attempts to cover/merge biological research with various fields of nanotechnology and entire basic scientific R\&D studying the fundamental, biological, physicochemical properties of nanomatters and biopolymer-protein assemblies, molecular motors, cellular electrochemical behaviours. Nano-biotechnology/bionanotechnology fantastically utilized chitin/chitosan: the $2^{\text {nd }}$ most natural polysaccharide just after cellulose in such perspectives [1,2]. Thus, varied formulations were done in chitin/chitosan matrix so as to use these bio-composites in clinical, biomedical and industrial fields as prominence like quantum/carbon dots, nanoparticle, nanocomposites and biosensors/biomarkers particularly for cancer detection. Nano-biotechnology exploited unique/admirable features of chitn/chitosan viz; biodegradability, biocompatibility, low/no toxicity, antimicrobial activity and low immunogenicity in drug delivery, siRNA/DNA delivery, tissue engineering, and wound healing, biosensors besides theranostics utilities Figure 1. Chitosan based nanobiotechnology is capably creates many novel nanomaterials/devices with vast applications and benefit to mankind. Strategic chitosan based nanoparticle carriers own vital impact on worldwide pharmaceutical marketing use to control drug release and get enhanced solubility of drugs, superior protein bioavailability, and better uptake of hydrophilic substances across epithelial layers besides great intracellular drug delivery [2-5].

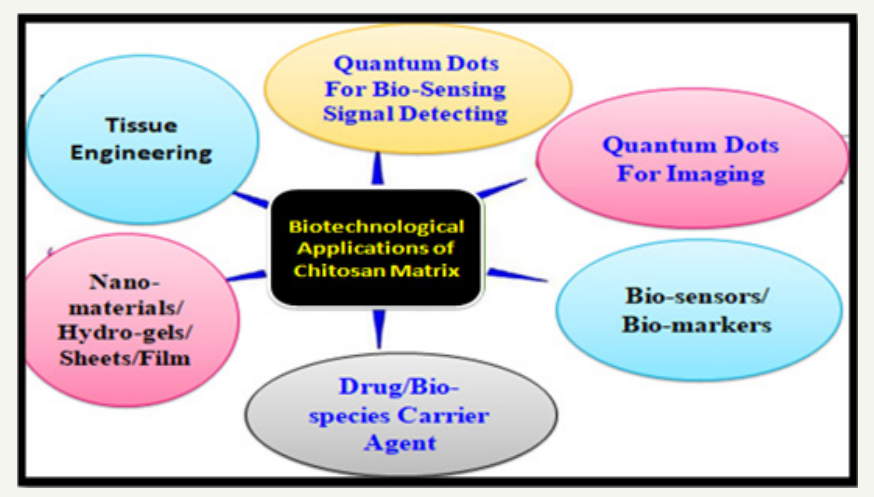

Figure 1: Varied bio-technology field applications of Chitosan Matrix.

\section{Nano-Biotechnologically Fabricated Chitin/chitosan Matrix}

\section{Chitosan -carbon dots}

Quantum dot are 'nanometre scale/zero-dimensional' semiconductor particles owing optical/electronic properties different than large particles and able to emit specific frequency of light/ray which can be tuned via nature of matter utilized, dot/ particle sizes, shapes and their arrangement. Because of extremely tuneable properties, brilliant water solubility, biocompatibility and better photo-stability the carbon base nano-chitonous quantum dots find empowered benefits in fluorescent bio-sensing/imaging [2-4]. Tea powder used to yield chitosan-carbon dots [2,3] as smooth, soft films which are robust to UV-visible blocking exploited in bio-medicals and imparted unique features like better swelled, thermal/mechanical properties compared to mere chitosan film with low cytotoxicity and excellent biocompatibility. Amino functionalized fluorescent carbon nitride dots own enhanced water solubility and strong fluorescent effect as advantageous in medical diagnosis and cancer treatment. Multi-colour chitosan-carbon dots exhibited bio-labeling potential varied bacterial model methods to use for bio-labeling in biomedicals [2-4]. Solid nano-solar cells from chitin, chitosan and glucose yields carbon quantum dot hybride with sensitizer nanozinc-oxide own utmost layer-by-layer coating competence of two kinds of carbon dots. Fluorescent nanocrystals/ quantum dots are used as an imaging agent for diseases detection own some limitations like toxicity and indiscretion thus novel zinc sulphide-chitosan quantum dots obtained with $\mathrm{pH}$ dependent/ tuneable optical/electrical properties to be used as probe in medicine/pharmaceuticals [2]. Luminescent chitosan-l-cysteine impregnated cadmium-tellurium films/dots exhibited antibacterial profile for broad range of biomedical utility. Chitosan-cadmiumtelluride based quantum dots generated onto indium-tin-oxide coated glass to be used for electrochemical biosensing of culprit DNA in chronic myelogenous leukemia/cancer detection. CdScarbon nanotubes and nano-gold-chitosan based quantum dots are used for formulating antibody immobilization owing brilliant control and bioactive profile compared to other immune-sensors for protein detection in medical studies [2-4]. Nano-chitosan formations have been developed for safe and effectual plasmid DNA, oligonucleotides and siRNA/gene carriers owing quick notice for gene delivery by treating silencing unwanted gene expression, defective gene and substituted missing genes in diseases curing therapeutics [1-5].

Chitosan-pEGFP nanoparticles are capable gene delivery vectors for an exogenous gene into primary chondrocytes own great potential to deliver therapeutic genes directly into joint to treat various types of joint diseases. Chitosan matrix modifications enhances transfection efficiencyvia self-branching eg., trisaccharidesubstitution yields linear counterparts with better gene transfer characteristics without negotiating biocompatibility for cellular uptake, and formulated stability [3-4]. N,N,N-trimethylated chitosan imparts better extracellular efficiency to promote intracellular siRNA release with good silencing activity and thus more appealing in vivo besides a capably imparts DNA-based drug delivery [2]. Glycol chitosan are useful nanocarriers to entrap chemotheraptics like doxorubicin/ DOX besides si-RNA with attenuated utmost efficiency via surmounts resistance in vivo distribution, adorn dose-dependent treatments [2-3]. Nanochitosan-poly-d,l-lactideco-glycolide are valuable nonviral device for enhanced pulmonary siRNA delivery besides in vitro gene silencing of in enhanced green fluorescent protein/endogenous H1299 cell line expression [2-5].

\section{Biosensor}

Sensor is a device used to receive and respond to signals besides converting into magnetic/electrical fields as further detected by an electronic device. The sensor utilized for the biological entities are termed as biosensor that coalesce natural component with a physicochemical detector to be analytically used for detection of 
sensitive biological species viz; tissue, microorganisms, organelles, cells, enzymes, antibodies, nucleic acids [1-5]. Biosensor yields via bio-mimetic way can be used for interaction/binding/recognition of analyte besides a diagnostic tool based on their specific biochemical interactive evaluation via DNA/RNA, enzymes, antibody cells and any signal transducer immobilized tissues [2-5]. Chitosan based biosensors are likely obtained via bio-engineered analytical gizmo that own beneficial usage due to prominent features like low cost, bio-compatibility and eco-friendly compared to others [4]. Analytical merits of chitosan based biosensor include adaptability, portability, high sensitivity, intrinsic selectivity and benign usage in moderately complex environments by virtue of quick responses [5]. Chitin/chitosan's flexibility provides immobilization of recognized species in various sensor gadgets as attractive matrixes for synthesis of enzyme sensors. These chitosan's parameters are exploited in fabrications of its nanocomposite biosensors for detection of assorted analyzes [2-5]. Tyrosinase-Fe304 doepd nano-chitosan biosensors are used for detection of organic phenols like catechol via porous chitosan matrix own large surface area as imparted by nano-iron oxide with high loaded tyrosinase enzyme for trapping pollutant [4-5]. Chitosan diffuse-graphene fabricates effectual biosensors for enzyme immobilization and glucose estimation with admirable sensitivity and long-term stability [35] due to large surface area and effective electrical conductivity features imparted by graphene. Chitosan- nanocarbon doped matrix yields amperometric biosensors use to encapsulate laccase besides utilized as biofuel cells, and other bio-electrochemical tools [5]. Nano-chitosan grafted polyaniline entrap creatinine amidinohydrolase onto electrochemically active surface with extraordinary immobilization of CAH enzyme and further assists to uphold the stability and durability.

\section{Biomarkers}

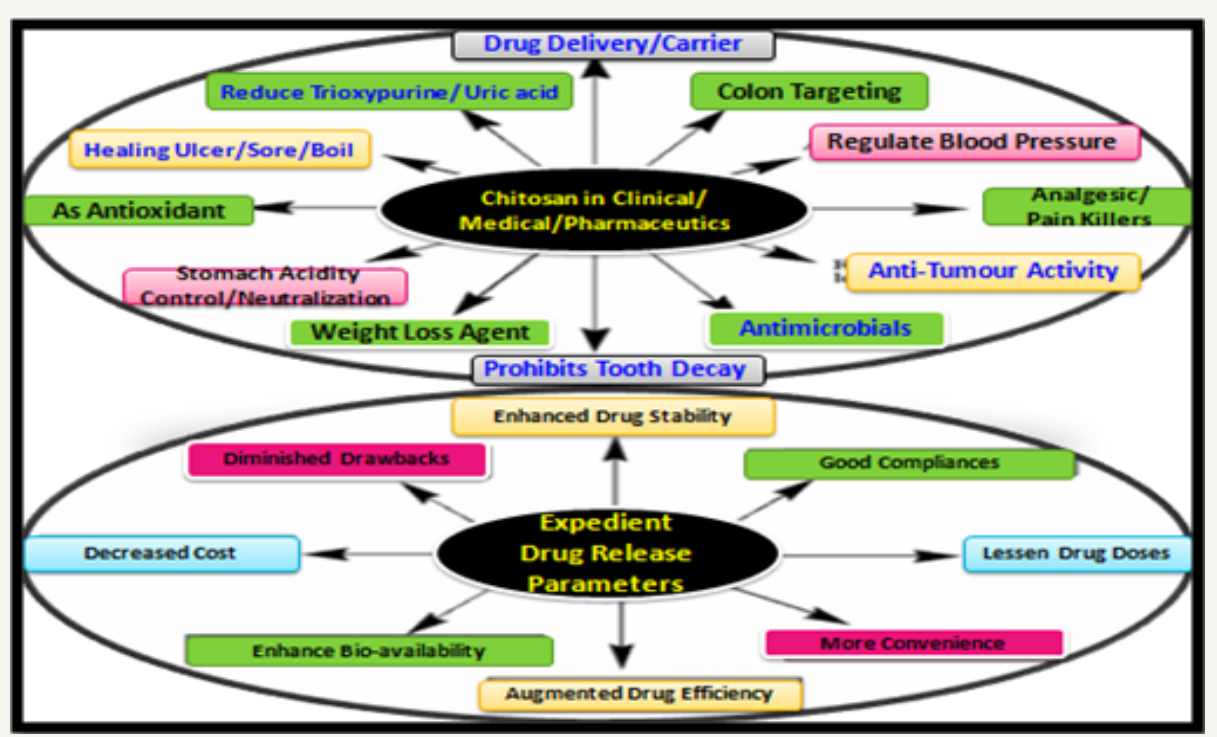

Figure 2: Pharmaceutical usages and expedient drug release parameters of chitosan.

Biomarker/biological marker measures some biological state/condition whose detection indicates the presence of a living organism. Biomarkers own characteristic objective measurement and evaluation/examination as an indicator of normal biological/ pathogenic processes, pharmacologic responses to a therapeutic intervention in assorted scientific fields besides to manage cancers and other diseases [2-5]. Biomarker practices in medical/clinical research field are routine and accepted almost without doubt [2]. Chitosan formulated biomarker are use to detect varied diseases due to expandable technical and huge clinical/medicinal significance roles like; facile screening and risk assessment before any disease diagnosis, can diagnose staging, grading, and initial therapy choices and monitor/modify the therapy or select additional therapy during treatment in certain cases [2-5] shown in Figure 2.

Chitosan fabricated gold coated polyelectrolyte multilayers/ xanthan own various biosensing and bio-imaging applications like detection of numerous diseases [2-4] and signal improvement for melanoma. Chitosan fabricated graphene/carbon nanospheres (CNS) imparts electrochemical immune-sensing marked with horseradish peroxidase-secondary antibodies for $\alpha$-fetoprotein induced cancer detections [2] as displayed in 7-fold signal increment compared to without graphene/CNS labeling immunosensors. An electrochemical biosensor/biomarkers based on chitosan nanocomposites used for detection of alphafetoprotein and carcinoembryonic antigen with more precise results than standard ELISA clinical diagnosis technique. Thus, chitin/chitosan integrated biomarkers have fascinated medical/ clinical applications with proficient personalized treatments and disease prevention. Chitin/chitosan own certain stupendous biological features like haemostatic, fungi-static, bacterio-static, spermicidal nature, anti-cholestermic and anti-carcinogenic which imparts vast significance in pharmaceutics including drug delivery, tissue engineering, gene delivery besides still to be explored for many other purpose (Figure 2). Primary $-\mathrm{NH}_{2}$ backbone of chitosan provides positive charge on its surface in acidic conditions besides 
its unique polycationic surfaces, capable inter/intra-molecular $\mathrm{H}$ - bonding, regarded it as best bio-matrix for the development of novel pharmaceutical/medical products. Bio-adhesiveness of chitosan provides adhesion to hard/soft tissues to be used in dentistry, orthopaedic, ophthalmology, surgical measures, optical and wave guiding properties [1-5].

\section{Limitations and Remedies}

Amongst all such advantages of chitn/chitosan, it also suffers from few drawbacks like being a weak base (pka=6.2) it own low at physiological/neutral $\mathrm{pH}$, mere soluble in organic acid-aqueous solution as glucosamine $-\mathrm{NH}_{2}$ gets protonated to form soluble salt $-\mathrm{NH}_{3}{ }^{+}$. Chitosan owes high swelling tendency in aqueous conditions resulted fast/quick drug release, thus needs to modify/tailored flexible chitosan skeleton. Certain demerits of chitosan can be overcome via 'derivatisation' performed either on primary amino/ $\mathrm{NH}_{2}$ or hydroxyl/OH groups of glucosamine polymeric units to resolve certain complications as per requirements for requisite utilities.

\section{Conclusion}

Chitosan are safe to be used in biomedicine, pharmacology and promising for development of harmless besides effective drug delivery systems. The mucoadhesive character of chitosan enhances residence time and consequently bioavailability of drug in target specific carriers. Fillers $\mathrm{ZnO}, \mathrm{ZnS}$ and $\mathrm{TiO}_{2}$ get intruding into chitosan matrix for widening the horizon of its utilities in biomedicals/ clinical yet not own much importance as pharmaceutical excipient due insufficient data regarding their mechanism and toxicity profiles to optimize its formulations. Hence, systematic research is anticipated to study chitosan polymeric interactions with added filler/dopent before and after drug delivery in biological systems which may open subsistence futuristic pharmaceutical usages.

\section{References}

1. Rajendra SD (2017) Biological activities and application of marine polysaccharide. Int-Tech Publisher Croatia 1: 181-206.

2. Tanvi J, Sushil K, PK Dutta (2015) Chitosan in the Light of Nanobiotechnology: A mini review. Journal of Biomedical Technology and Research 1(1): 101-107.

3. Bănică, Gabriel F (2012) Chemical sensors and biosensors: fundamentals and applications. Chichester, John Wiley \& Sons. UK, p. 576.

4. Jianc H, Su W, Caracci S, Bunninc TJ (1996) Optical waveguiding and morphology of chitosan thin films. J Appl Polym Sci 61(7): 1163-1171.

5. Burkatovskaya M, Tegos GP, Swietlik E, Demidova TN, P Castano A, et al. (2006) Use of chitosan bandage to prevent fatal infections developing from highly contaminated wounds in mice. Biomaterials 27(22): 41574164. 